\title{
Reflections on a recent report of the World Anti-Doping Agency
}

\author{
Nicolaas M. Faber
}

Accepted: 21 January 2014/Published online: 13 February 2014

(C) Springer-Verlag Berlin Heidelberg 2014

Sir,

Criticism on the test results delivered by doping control laboratories often meets a response by researchers acting on behalf of the World Anti-Doping Agency (WADA) that essentially amounts to "we know best what needs to be done." A noteworthy example of such criticism is the thought-provoking Commentary in Nature, entitled "The science of doping" [1] which calls for further developments in the related analytical science and improvements in its application.

Recently, an unprecedented development shows that this kind of criticism may be well founded in current antidoping practice. Last May, WADA published a report detailing why current testing as performed in several laboratories has weaknesses [2]. I was absolutely stunned to encounter the following deficiencies in an even much longer list:

1. It is much simpler for laboratories to err on the side of negative reporting than to report positive samples and then risk having to defend their analysis or to be exposed to public scrutiny;

2. Not all accredited laboratories are capable of operating at the same level of competence;

3. Not all laboratories practice at recognized ISO quality management levels or even follow the ISL [International Organization for Standardization, International Standard for Laboratories].

N. M. Faber $(\bowtie)$

Chemometry Consultancy, Goudenregenstraat 6,

6573 XN Beek-Ubbergen, The Netherlands

e-mail: nmf@chemometry.com
Let me limit myself to three major observations:

1. From point 1 , one could assume that some laboratories allow for false negatives. In doing so, they essentially take the seat of the judge.

2. From points 2 and 3, one can infer that the test result may depend on the laboratory. Indeed, this is well known for clenbuterol, for example. The Spanish cyclist Alberto Contador had the 'bad luck' of his samples of the Tour de France 2010 being analyzed in the doping control laboratory of Cologne, resulting in the loss of his title. He may have been tested 'negative' in another laboratory.

3. A laboratory not following the ISL (see point 3) presents an enormous risk for both (innocent) athletes and (prosecuting) sports organizations. A case in point of the latter is the needless loss of a case by the International Olympic Committee [3], because the deviation from the ISL could be demonstrated.

It stands to reason that elite sport deserves elite testing. WADA now acknowledges that the expected level of testing is not always delivered by WADA-accredited laboratories, owing to weaknesses in following strict quality management procedures and protocols. In conclusion, it appears highly desirable that researchers stay open to criticism, when it is clearly meant to be constructive. More generally speaking, constructive criticism can be seen as free advice.

\section{References}

1. Berry DA (2008) Nature 454:692-693

2. http://www.wada-ama.org/Documents/World_Anti-Doping_Program/ Reports-Assessments/2013-05-12-Lack-of-effectiveness-of-testingWG-Report-Final.pdf

3. http://www.tas-cas.org/d2wfiles/document/4277/5048/0/Award20 Devyatovskiy20\&20Tsikhan20internet.pdf 